\title{
FORMACIÓN DE RECURSOS HUMANOS EN EL SECTOR AGROPECUARIO Y AGROINDUSTRIAL A TRAVÉS DE LA ARTICULACIÓN DE EDUCACIÓN SUPERIOR, MEDIA, NO FORMAL Y SECTOR PRODUCTIVO
}

\section{Introducción}

Los desafíos que surgen a partir de los tratados y acuerdos comerciales que asume el país, definido como abierto al mercado y al fomento de las exportaciones, abren las puertas a una serie de oportunidades para diversos rubros agropecuarios y agroindustriales, y a otras alternativas muy poco exploradas aún, como son la producción orgánica o productos nativos.

Los requerimientos técnicos de la producción agropecuaria y agroindustrial obligan a trabajar en un ambiente de Good Manufacturing Practices (GMP), o buenas prácticas de manufactura, y ampliar el horizonte a las BPA (buenas prácticas agrícolas), asunto que requiere una sensibilización y entrenamiento de quienes llevan a cabo la producción y comercialización, aspectos definidos por el Estado como áreas estratégicas para el país. Finalmente, las normas ISO 9000 (Gestión de Calidad), ISO 14000 (Gestión Medioambiental) e INN 18000 (Seguridad de los Trabajadores) deberán ser aplicadas en forma masiva si se desea homologar la normativa europea.

La formación de recursos humanos en el sector agropecuario resulta de suma importancia, más aún si se estima que, regionalmente, este sector ha mostrado un crecimiento económico bajo el promedio nacional. América Latina genera alrededor del 8,4\%

1 International Organization for Standardization. 
del PIB nacional regionalizado (Banco Central, 2003). Dentro de la estructura productiva de la región predomina la rama industrial, luego el sector servicios y, en tercer lugar, se ubica la agricultura (incluyendo silvicultura y caza), aportando aproximadamente el $10 \%$. En cuanto al empleo, en primer lugar se ubican los servicios comerciales y sociales, luego la rama industrial, con un $13,5 \%$, y, en tercer lugar, el sector agrícola que genera un $12,5 \%$. A su vez, presenta transformaciones que se manifiestan de manera diferente en distintos tipos de productores; sin embargo, en general, se observa una significativa disminución de la superficie destinada a cultivos tradicionales, como cereales, leguminosas viñas y algunos cultivos industriales (remolacha y maíz), acompañada de importantes aumentos de rendimientos. Los rubros asociados a mayores niveles de procesamiento han mantenido o aumentado la superficie cultivada, lo que se explica por las modificaciones en el escenario económico de las últimas décadas (Fawaz, 2004).

Uno de los requerimientos que debe enfrentar el sector, a la brevedad, es contar con recursos humanos altamente calificados, capaces de incorporar nuevas tecnologías a la producción, con competencias que le permitan actuar en forma efectiva en un nuevo y dinámico campo laboral y con habilidades cognitivas y técnicas para desarrollar una formación continua y permanente.

Sin embargo, estos requerimientos contrastan con los niveles de educación que actualmente presenta el sector agropecuario. Si consideráramos a quienes no tienen ninguna escolaridad (12\%) y a quienes tienen educación básica incompleta (53\%), queda de manifiesto la carencia de información adecuada para la gestión y la baja capacidad de destreza analítica para competir eficazmente e incorporarse en las cadenas de valor agregado (Fonck, 2002). 
Gráfico 1

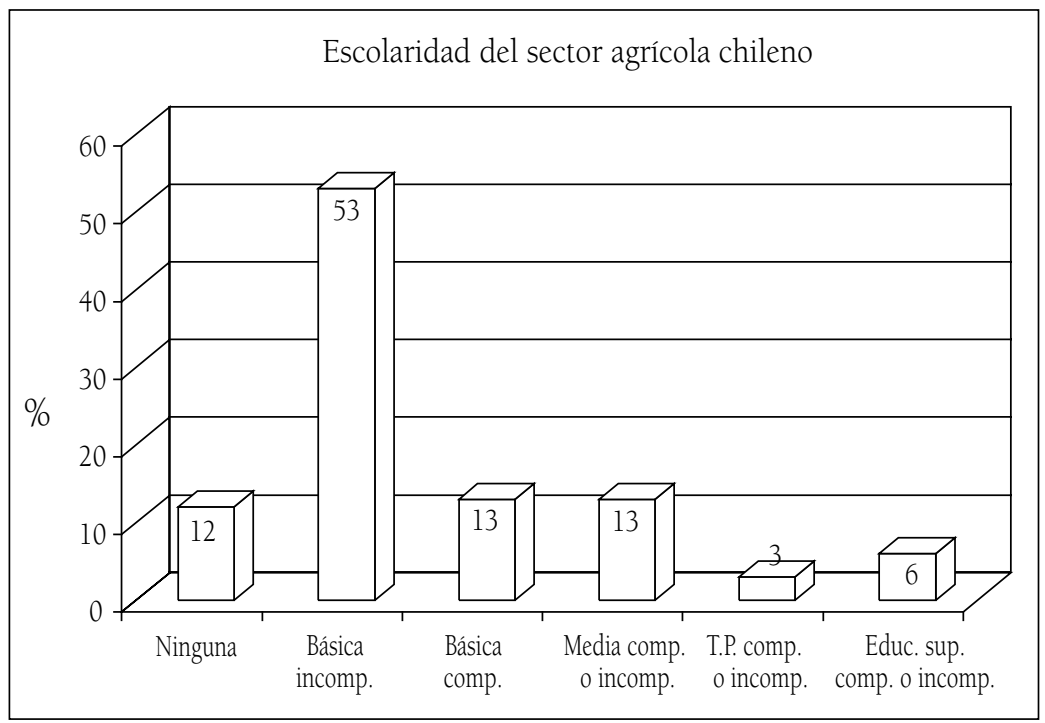

\section{Alianzas para la formación de recursos humanos en el sector}

Considerando esta realidad, y la alta dispersión geográfica de los productores agropecuarios, se constituyó, en la Región del Bío Bío, en el marco del programa Chilecalifica, una alianza con el objetivo de contribuir a la generación de un sistema de formación continua y articulada, que permitiera potenciar las competencias de actitudes personales, sociales, productivas y competitivas, para optimizar la producción, la agregación de valor y la gestión y comercialización, considerando el ámbito de equidad social, de sustentabilidad ambiental y el sentido de identidad rural ${ }^{2}$.

La alianza entre instituciones de educación superior, media y el sector productivo permitió la constitución de una red de articulación de la formación agropecuaria, en la que participan instituciones que imparten educación agropecuaria y agroindustrial

2 Proyecto de articulación y mejoramiento de la formación agropecuaria y agroindustrial de la VIII Región. Red Agroforma-Chilecalifica 2003. 
en sus distintos niveles: universidades, institutos profesionales, centros de formación técnica, liceos técnicos agropecuarios y agroindustriales y organismos técnicos de capacitación, junto con empresas y asociaciones gremiales productivas, que representan y demandan las competencias requeridas en los distintos ámbitos sectoriales ${ }^{3}$.

Tabla 1. Sector formativo y productivo integrante de la red agropecuaria y agroindustrial Red Agroforma de la Región del Bío Bío

\begin{tabular}{|l|c|}
\hline Sector formativo & Integrantes \\
\hline Universidades & 3 \\
\hline Institutos profesionales & 2 \\
\hline Centros de formación técnica & 1 \\
\hline Liceos de educación media técnico-profesional agropecuarios & 9 \\
\hline Liceo de educación media técnico-profesional agroindustriales & 1 \\
\hline Liceo de educación de adultos & 1 \\
\hline Organismos técnicos de capacitación & 4 \\
\hline Sector productivo & 0 \\
\hline Empresas agropecuarias y agroindustriales & 7 \\
\hline Federación de organizaciones de productores agropecuarios & 1 \\
\hline Asociaciones gremiales de productores agropecuarios & 4 \\
\hline Cooperativa de productores agropecuarios & 1 \\
\hline Total & 34 \\
\hline
\end{tabular}

Fuente: Convenio de cooperación, integrantes de Red Agroforma. 2003

Esta alianza ha permitido avanzar en la creación de un modelo de formación continua en el ámbito técnico, a través del proyecto "Articulación y mejoramiento de la formación técnicoprofesional agropecuaria y agroindustrial de la región del Bío Bío", en el marco del programa Chilecalifica ${ }^{4}$. El modelo busca responder a las necesidades del desarrollo productivo agropecuario regional y nacional. De acuerdo con los lineamientos del programa, requiere

3 Informe anual del proyecto de articulación y mejoramiento de la formación agropecuaria y agroindustrial de la VIII Región. Red Agroforma-Chilecalifica 2004.

4 Proyecto de articulación y mejoramiento de la formación agropecuaria y agroindustrial de la VIII Región. Red Agroforma-Chilecalifica 2003. 
de una articulación vertical de distintos niveles de formación; de una horizontal, a través de la estructuración de un sistema de reconocimiento, validación y certificación de competencias adquiridas de manera formal, informal o en el trabajo realizado, y, en forma complementaria, de un sistema que permita la formación de un agricultor profesional, a partir de las instancias actuales de formación y otras que sea necesario implementar.

\section{Modelo de articulación}

La red ha desarrollado un modelo en el cual cada uno de sus integrantes tiene desafíos; corresponde a las universidades que participan el papel de preparar a los profesionales que se desempeñan en los diferentes niveles de formación, para que obtengan las competencias necesarias que les permitan, a su vez, estructurar su acción capacitadora sobre la base de competencias.

El modelo considera la participación activa del sector productivo, a través de la definición de las competencias laborales requeridas; ello permite proponer ajustes en los perfiles de egreso de los técnicos de nivel medio y superior, de acuerdo con la realidad sectorial. La información desde el sector productivo se ha obtenido mediante el análisis situacional del trabajo (AST), en rubros priorizados en los Planes de Desarrollo Regional y validado a través de talleres de trabajo con representantes del sector productivo, razón por la cual esta información no sólo es de utilidad para proponer ajustes a los perfiles de egreso de los niveles medios y técnico superior, sino que también para las universidades, ya que es un insumo para adecuar planes y programas considerando esta realidad.

Para complementar las competencias no alcanzadas, el modelo considera la implementación de módulos de formación básica o de mayor especialidad, los que deberán ser asumidos por las instituciones de formación, en sus distintos niveles, según requerimientos. 
Finalmente, propone estructurar un currículo que permita formar un agricultor profesional, capaz de participar de manera competitiva en las actuales condiciones del sector. Este currículo considera una formación básica general y una de especialidad, según la orientación productiva del agricultor. Se accede a él a través del reconocimiento de las competencias previas y adquiriendo las competencias requeridas en módulos del sistema de capacitación existentes o generadas por las instituciones de formación participantes. En este aspecto, las instituciones de educación superior deben asumir la preparación de los equipos de formadores que concretarán la propuesta, no sólo en los aspectos de conocimiento y tecnología sino que, también, en las actitudes que se requieren para el adecuado desempeño en la producción agropecuaria y agroindustrial.

Modelo de articulación para la formación técnico-profesional agropecuaria y agroindustrial de la Región del Bío Bío, propuesto en el marco del

programa Chilecalifica

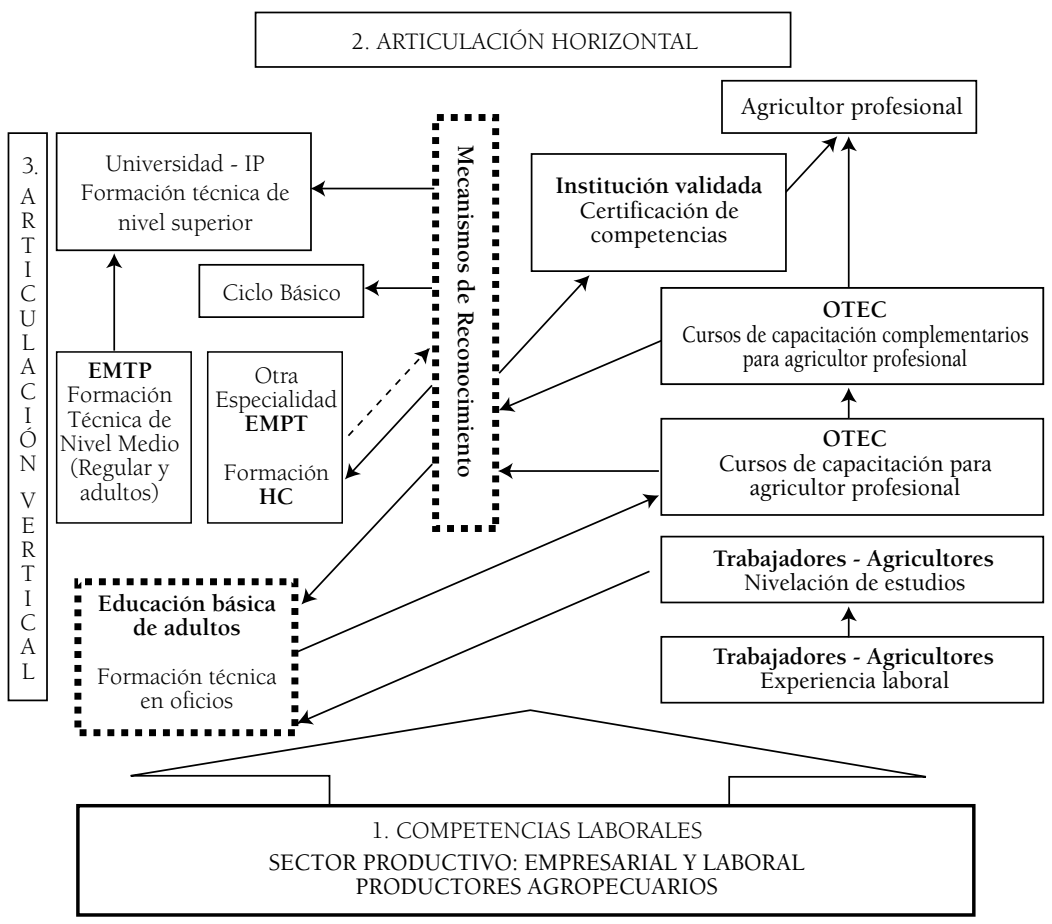




\section{Sistema de reconocimiento de competencias}

En este sector no existen mecanismos que permitan el reconocimiento adecuado de competencias técnicas adquiridas previamente, por lo que la red, con el aporte activo de las universidades que la integran, está abocada a la tarea de diseñar instrumentos de reconocimiento, validación y certificación de competencias adquiridas, de manera informal o a través de la experiencia laboral, lo que facilitará la articulación vertical y horizontal de la formación, y que las personas puedan acceder a una capacitación de nivel superior reduciendo tiempo y, por ende, costos.

Al analizar la participación del sector productivo, se observa que, si bien requiere mejorar la competitividad de sus trabajadores y que ella sea validada, tiene también el desafío de generar las condiciones para que a quienes mejoren su competencias laborales les sea reconocido su esfuerzo y se les asignen tareas correspondientes a su nueva condición, estimulando la actitud hacia la formación permanente.

Principales logros de la red:

- Articulación de distintas instituciones educativas y gubernamentales, organizaciones y empresas del sector.

- Propuesta de ajuste a perfiles de egreso del nivel medio y técnico superior, y su correspondiente articulación.

- Fortalecimiento de la presencia de las universidades en el sector productivo regional.

- Mejoramiento de las condiciones de docencia de los establecimientos de nivel medio asociados a la red y de la autoestima del personal relacionado con esta labor.

- Contribución a la visibilidad del sector agropecuario y agroindustrial regional. 


\section{Consideraciones generales}

Un imperativo que debe ser asumido de manera integral, para que el modelo se aplique en plenitud, es generar las condiciones para que los alumnos de educación técnico-agrícola y agroindustrial de nivel medio en la región continúen en el nivel superior en un centro de formación técnica (CFT). El liceo agrícola cuenta con sistema de internado subvencionado por el Estado, lo que hasta la fecha no existe en los CFT, y los estudiantes deben asumir los costos que implica proseguir sus estudios. Al respecto, la ampliación de becas con respaldo estatal se ve como un paso favorable para reducir la brecha de desigualdad que esta situación produce.

\section{Referencias bibliográficas}

Banco Central. Producto Interno Bruto Regional 2002-2003, Santiago de Chile, 2003.

Fawaz, M.J. y Silva, A.M. La inserción de las economías rurales en el proceso de globalización. El caso de la provincia de Ñuble en Chile. Buenos Aires, 2004, pp. 5-9.

Fonck, O.C. y Oyarzún, L.L. Formación del agricultor profesional. La apuesta de Chile para competir en un mundo globalizado. IICA, Santiago de Chile, 2002, pp. 31-33.

Ministerio de Agricultura. Protocolo de acuerdo. Agenda de dinamización del sector silvoagropecuario en los territorios de la Región del Bío Bío. Concepción, junio 2004.

Red Agroforma 2003. Proyecto de articulación y mejoramiento de la formación agropecuaria y agroindustrial de la VIII Región, Chillán, 2003. 